INPLASY

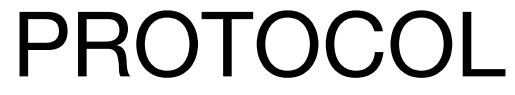

To cite: Lee et al. Risk

Prediction Models for Hospital

Readmission: An Update of a

Systematic Review. Inplasy

protocol 202090099. doi:

10.37766/inplasy2020.9.0099

Received: 27 September 2020

Published: 28 September 2020

Corresponding author:

Itong Lee

liyitongr@gmail.com

Author Affiliation:

The Capital Medical University, China

Support: None.

Review Stage at time of this submission: Preliminary

searches.

Conflicts of interest:

Have no known conflicts of interest.

\section{Risk Prediction Models for Hospital Readmission: An Update of a Systematic Review}

Lee, YT1; Liu, Y².

Review question / Objective: How does risk prediction models perform on predicting of patients' hospital readmission compared to models developed ten years earlier?

Condition being studied: Hospital Readmission, Risk prediction model There is an increasing trend of making hospital readmission prediction models, especially during this era of artificial intelligence. But not all of these new models are of great necessity. Some are doing repeat works, while some have poor quality. But it has been clear that predicting patients' readmission as early as possible is good to both society and patients. We want to find out which risk factor matters most and how these models perform.

INPLASY registration number: This protocol was registered with the International Platform of Registered Systematic Review and Meta-Analysis Protocols (INPLASY) on 28 September 2020 and was last updated on 28 September 2020 (registration number INPLASY202090099).

\section{INTRODUCTION}

Review question / Objective: How does risk prediction models perform on predicting of patients' hospital readmission compared to models developed ten years earlier?
Rationale: Our study is an update systematic review, based on the article: Risk Prediction Models for Hospital Readmission -- A Systematic Review, which published on October 19, 2011. It has been ten years since the review published, while the huge social financial burden from 
hospital readmission and the poor prognosis of readmission patients still exist. During the ten years, plenty of new models developed and validated, and some of them might provide us new ideas. So, we think it is necessary to update the prediction models and try to find some trends and possible solutions for both clinical and administrative use.

Condition being studied: Hospital Readmission, Risk prediction model There is an increasing trend of making hospital readmission prediction models, especially during this era of artificial intelligence. But not all of these new models are of great necessity. Some are doing repeat works, while some have poor quality. But it has been clear that predicting patients' readmission as early as possible is good to both society and patients. We want to find out which risk factor matters most and how these models perform.

\section{METHODS}

Search strategy: Include but not limited to: Terms: Readmission, Risk, model, Predict, Probability of repeated admissions Databases: MEDLINE, Embase, CINAHL, PsycINFO, Cochrane.

Participant or population: Adult patients admitted to a medical service. Postsurgical patients and psychiatric readmissions are excluded.

Intervention: Risk prediction models derived and validated in a cohort of medical inpatients.

Comparator: Studies comparing the performance of two or more risk prediction models in a population will be included.

Study designs to be included: Risk prediction models derived and validated in a cohort of medical impatients.

Eligibility criteria: Exclude studies conducted in health systems of developing nations.
Information sources: Sources include (but are not limited to) bibliographic databases, reference lists of eligible studies and review articles, key journals, conference proceedings, trials registers, Internet resources and contact with study investigators, experts and manufacturers. Search dates: From July, 2011 to July, 2021; Restrictions on search: Language in English; Searches will be rerun prior to the final analysis.

Main outcome(s): Hospital readmission including all-cause readmissions, condition-specific readmissions, and potentially preventable readmissions. Readmission of inpatients to ICU is excluded.

Quality assessment / Risk of bias analysis: The c statistic with $95 \%$ confidence intervals (when available) were used to describe model discrimination. If the $c$ statistic was not reported, we abstracted other operational statistics such as sensitivity, specificity, and predictive values for representative risk score cutoffs when available. To describe model calibration, we report the range of observed readmission rates from the predicted lowest to highest risk groupings.We adapted elements (including cohort definition, follow-up, adequacy of prognostic and outcome variable measurement, and the validation method) from a prognosis study quality tool and clinical decision rule assessment tool.

Strategy of data synthesis: The included studies were too heterogenous to permit meta-analysis. Therefore, we qualitatively synthesized results, focusing on model discrimination, the populations in which the model has been tested, practical aspects of model implementation, and the types of variables included in each model.

Subgroup analysis: We qualitatively synthesized results instead of doing subgroup analysis, as I stated above. For our main purpose was to compare models and try to find relatively better ones, and summarize their characteristics in common. 
Sensibility analysis: We prefer to use c statics to describe the models unless the article did not provide us. In this case, we shall use sensibility analysis, or specificity, predictive values for representative risk score cutoffs when available.

Country(ies) involved: China.

Keywords: Risk factors, Prediction Models, Hospital readmission, Update.

Contributions of each author:

Author 1 - Itong Lee.

Author 2 - Yan Liu. 